\title{
Linear polarization of pulsar radio emission
}

\author{
M. Gedalin and Y. Dzigan
}

\author{
Ben-Gurion University, 84105 Beer-Sheva, Israel \\ e-mail: gedalin@bgumail.bgu.ac.il
}

Received 5 January 2005 / Accepted 8 April 2005

\begin{abstract}
We suggest that the observed linear polarization of pulsar radio emission is due to features of the emission mechanism that produce electromagnetic waves in a narrow cone around a magnetic field line. As a result, emission collected along the line of sight does not come from a single magnetic field line but from many non-parallel lines, each of which acts on an elementary emitter to generate a single linearly polarized mode. The combined emission from the bunch of rays should be nearly unpolarized at the emission site. When the rays propagate outward, the polarization of the individual ray follows that of the normal mode until the polarization limiting radius (PLR) is reached. The resulting degree of linear polarization will depend on the details of the magnetic field distribution across the emitting bunch at the PLR. We show that for the model of Gedalin et al. the PLR is deep in the magnetosphere, and we demonstrate how the linear polarization forms.
\end{abstract}

Key words. pulsars: general - polarization - plasmas - radiation mechanisms: non-thermal

\section{Introduction}

Among the puzzles of pulsar radio emission the polarization properties have an important place. The radio emission is, as a rule, partially linearly polarized and often circularly polarized, while the polarization is more pronounced in single pulses than in the averaged emission (Stinebring et al. 1984a,b; Karastergiou et al. 2001, 2002; Kramer et al. 2002), and increases with finer time resolution (Gangadhara et al. 1999). This observed behavior of the polarization motivated the orthogonal polarization mode (OPM) models (Cordes et al. 1978; McKinnon \& Stinebring 1998; McKinnon 2003), with the strong emphasis on the superimposed OPM version (Karastergiou et al. 2002) where the two orthogonal modes exist simultaneously in the observed radio emission, and the observed features are related to the relative strength of the modes and their correlation (McKinnon \& Stinebring 1998). Since all plausible proposed mechanisms of pulsar radio wave generation imply that only one linearly polarized mode is produced (see, e.g. Gedalin et al. 2002a,b), substantial efforts have been devoted to explain how the second mode or elliptical addition can be produced from propagation effects in the magnetospheric plasma (see, e.g. Lyubarskii \& Petrova 1998; Petrova 2001, and references therein). In all these models the radiation propagating along the line of sight of an observer is initially completely polarized, and depolarization is achieved via production of the other orthogonal mode. It is implicitly assumed that the emission comes along a single ray generated by a single source, so that the initial polarization is completely determined by the magnetic field direction at this point. However, it is highly unlikely that a one-dimensional distribution of radio waves is generated at the emission sight. It is much more likely that this distribution has a certain spatial width, in which case the initial distribution may be produced by more than one elementary source (see also the recent pioneering work in this direction by Luo 2004), thus making the combined radio emission substantially depolarized from the very beginning. Below we show that in this case the emission becomes progressively more linearly polarized as it propagates outward along the line of sight. The resulting polarization is determined by the shape of the viewed emission region at the polarization limiting radius (PLR), beyond which the electric vector of a propagating wave no longer follows the polarization direction prescribed by the normal mode features.

If a single linear polarized mode is generated and propagates along a ray (coming from a single point-like source), its polarization corresponds to that of the normal mode until mode coupling becomes significant (Melrose, private communication) or the wave properties are no longer affected by the medium (plasma). Mode coupling may give rise to a second orthogonal mode, so that the radiation propagates further as a mixture of two normal modes. Mode coupling is expected near a radius $r_{\mathrm{m}}$ where the relative difference between the wavenumber of the two orthogonal normal modes, $\Delta k$, becomes comparable to the change of their polarization due to inhomogeneities in the plasma, $\Delta k L \ll 1$, where $L$ is the typical length of the inhomogeneity (Melrose \& Stoneham 1977; Cheng \& Ruderman 1979; Barnard \& Arons 1986; Lyubarskii \& Petrova 1998). Existing estimates of the depolarization due to this mode coupling are small (see, e.g., Petrova 2001). The radio waves 
propagate outward into the lower density regions. For the electromagnetic waves in a relativistic pulsar plasma the phase difference for the two modes is $\Delta \psi=\int \Delta k \mathrm{~d} l=\int \omega \Delta n \mathrm{~d} x / c$, where $|\Delta n| \sim|n-1| \ll 1$ and rapidly decreases as a function of the distance from the pulsar. This phase difference becomes insignificant at the radius $r_{\mathrm{p}}$ such that $\int_{r_{\mathrm{p}}}^{\infty} \Delta k \mathrm{~d} l \lesssim 1$. Moreover, within the above assumptions, $\int_{r_{\mathrm{p}}}^{\infty}|k-\omega / c| \mathrm{d} l \lesssim 1$, so that beyond this radius the deviation of the wave from the vacuum electromagnetic wave becomes negligible, and further polarization changes do not occur. This radius is commonly referred to as the polarization limiting radius (PLR) (Melrose \& Stoneham 1977; Cheng \& Ruderman 1979; Barnard \& Arons 1986; Petrova 2001). If $r_{\mathrm{m}}<r_{\mathrm{p}}$ mode coupling may be expected to noticeably affect the observed radiation polarization by adding another normal mode. If, however, $r_{\mathrm{p}}<r_{\mathrm{m}}$, no substantial mode coupling occurs and the observed polarization will be the polarization of the originally emitted normal mode at the PLR.

The paper is organized as follows. In Sect. 2 we provide a brief description of the polarization for the emission coming from multiple sub-sources. In Sect. 3 we analyze what happens when radio waves, which are originally emitted at small radii, propagate to the PLR. We follow the model by Gedalin et al. (2002a,b) and show that the PLR is typically closer to the emission zone than is the mode coupling radius, $r_{\mathrm{p}}<r_{\mathrm{m}}$. The last relation allows us to qualitatively estimate the polarization patterns.

\section{Polarization for multiple sub-sources}

If the observed radiation comes from a single ray, the features of this radiation will be completely determined by the behavior of this single ray at the polarization limiting radius. In order to account for the observed emission (which is not completely polarized), a wave, initially completely linearly polarized, would have to be substantially depolarized when crossing the PLR site, that is, a sufficiently large incoherent second orthogonal component would have to appear in addition to the the original electric field. Existing calculations (see, e.g. Petrova 2001) produce changes of the polarization but the arising components are still small and further depolarization is necessary to make the two components incoherent. On the other hand, if the observed radiation comes from many essentially incoherent sources (Luo 2004), the resulting polarization would be determined by the preferential polarization of the these sources.

Let us make a very simple estimate of the Stokes parameters of the emission, which is an incoherent superposition of emissions coming from many individual sources. In order to make the analysis simple, retaining basic physics but getting rid of unnecessary complications, let us assume that each single source $i$ contributes the electric field vector of

$\boldsymbol{E}_{i}=E\left[\hat{\boldsymbol{e}}_{i}+\mathrm{i} \mu\left(\hat{\boldsymbol{n}} \times \hat{\boldsymbol{e}}_{i}\right)\right] \exp \left(\mathrm{i} \phi_{i}\right)$,

where the amplitude $E$ is the same for all sources, $\hat{\boldsymbol{e}}_{i}$ is the direction of the main polarization axis, $\hat{\boldsymbol{n}}$ is the direction of the line of sight, $\hat{\boldsymbol{n}} \perp \hat{\boldsymbol{e}}_{i}$, and $\phi_{i}$ is the phase. The parameter $\mu$, characterizing the degree of elliptical polarization, is also assumed to be the same for all single rays. The assumptions of $E=$ const. and $\mu=$ const. are not necessary but make the analysis more transparent. The resulting electric field is $\boldsymbol{E}=\sum_{i} \boldsymbol{E}_{i}$ and the polarization matrix would take the form

$$
\begin{aligned}
P_{\alpha \beta}= & \left\langle E_{\alpha} E_{\beta}^{*}\right\rangle \\
= & |E|^{2}\left[\left\langle e_{\alpha} e_{\beta}\right\rangle+\mu^{2}\left\langle(\hat{\boldsymbol{n}} \times \hat{\boldsymbol{e}})_{\alpha}(\hat{\boldsymbol{n}} \times \hat{\boldsymbol{e}})_{\beta}\right\rangle\right. \\
& \left.+\mathrm{i} \mu\left\langle(\hat{\boldsymbol{n}} \times \hat{\boldsymbol{e}})_{\alpha} e_{\beta}-(\hat{\boldsymbol{n}} \times \hat{\boldsymbol{e}})_{\beta} e_{\alpha}\right\rangle\right],
\end{aligned}
$$

where $\langle\cdots\rangle$ denotes averaging, and we assumed that the phases are independent, $\left\langle\exp \left[\mathrm{i}\left(\phi_{i}-\phi_{k}\right)\right]\right\rangle=\delta_{i j}$. Defining for convenience the polar angle $\chi$ in the plane perpendicular to $\hat{\boldsymbol{n}}$, so that $\hat{\boldsymbol{e}}=(\cos \chi, \sin \chi)$ and $(\hat{\boldsymbol{n}} \times \hat{\boldsymbol{e}})=(\sin \chi,-\cos \chi)$, we arrive at the following matrix:

$P=|E|^{2}\left(\begin{array}{cc}\left\langle\cos ^{2} \chi\right\rangle+\mu^{2}\left\langle\sin ^{2} \chi\right\rangle & \langle\sin \chi \cos \chi\rangle-\mathrm{i} \mu \\ \langle\sin \chi \cos \chi\rangle+\mathrm{i} \mu & \left\langle\sin ^{2} \chi\right\rangle+\mu^{2}\left\langle\cos ^{2} \chi\right\rangle\end{array}\right)$

so that the Stokes parameters are (normalized on $|E|^{2}$ for convenience):

$$
\begin{aligned}
I & =1+\mu^{2}, \quad Q=\left\langle\left(\cos ^{2} \chi-\sin ^{2} \chi\right)\right\rangle, \\
U & =\langle\sin \chi \cos \chi\rangle, \quad V=\mu .
\end{aligned}
$$

Let us assume that $\mu \ll 1$ and that $\chi$ are uniformly distributed in the range $-\chi_{0}<\chi<\chi_{0}$. Then the degree of linear polarization would be $\Pi_{L}=\sin \left(2 \chi_{0}\right) / 2 \chi_{0}$, while the degree of circular polarization is $\Pi_{V}=\mu$. For $\chi_{0}=\pi / 4$ one finds $\Pi_{L}=$ $2 / \pi \approx 64 \%$. The polarization angle $\xi=(1 / 2) \arctan (U / Q)$ may be well determined even if the linear polarization degree $\Pi_{L}=\left(Q^{2}+U^{2}\right)^{1 / 2} / I$ is small.

Thus, the narrow cone emission (instead of a single ray) can, in principle, explain the partial linear polarization of the pulsar radio emission. Quantitative results depend on the plasma parameters in the pulsar magnetosphere.

\section{The PLR}

The observed polarization is determined by the distribution of viewed sub-sources at the PLR, beyond which the wave polarization no longer changes. If the observed emission consists of a number of parallel rays coming from a number of independent sources, the polarization properties depend on the polarizations in all these rays at the PLR. The model presented by Gedalin et al. (2002a,b) proposes that radio waves are generated by the beam instability in the pulsar plasma deep in the magnetosphere. The waves are produced within the angle $\theta_{0} \sim 1 / \gamma_{\mathrm{b}}$ around the local magnetic field direction (field line), where $\gamma_{\mathrm{b}}$ is the Lorentz-factor of the beam. These waves decouple from the beam at the radius $R_{0}$ where $2 \omega_{\mathrm{p}}^{2} \gamma \approx \omega^{2}$, where $\gamma$ is the Lorentz-factor of the plasma body. Thus, at the emission site the rays from the "spot" of the radius $L_{0} \sim R_{0} \theta_{0} \sim R_{0} / \gamma_{\mathrm{b}}$ are initially propagating in the same direction. This "spot" consists of a number of independent microsources if $k_{\perp} L_{0} \gg 1$, where $k_{\perp} \sim \omega \theta_{0} / c$ is the typical perpendicular wave number of the waves (wave number uncertainty). Thus, the initial source is not a point but a large number of elementary sources if

$\omega R_{0} / c \gamma_{\mathrm{b}}^{2} \gg 1$. 


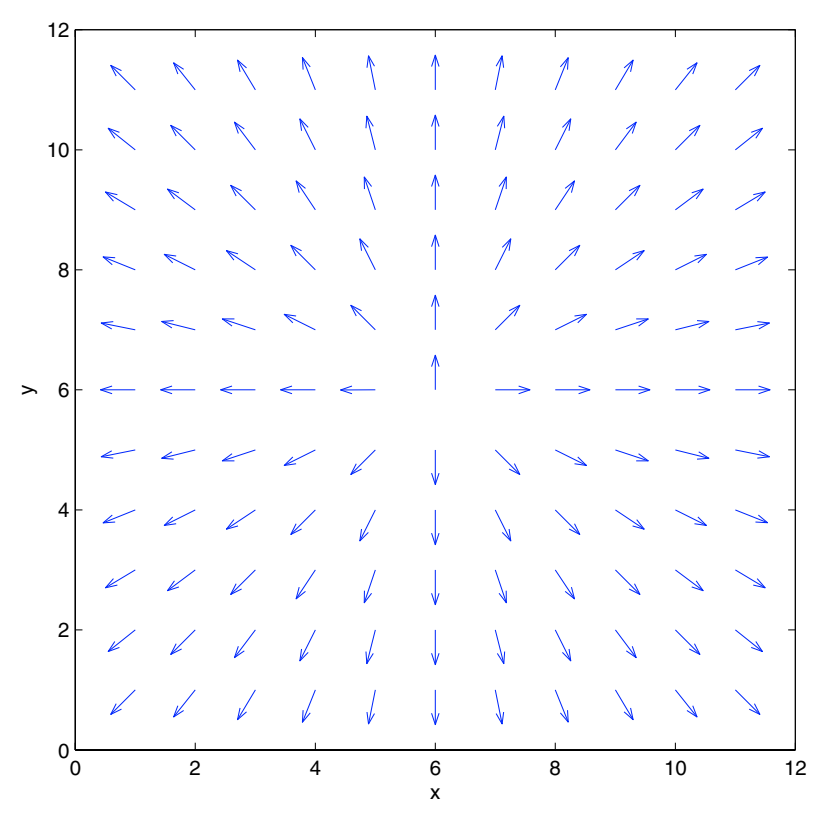

Fig. 1. Projections of the magnetic field vector at the emission site - "spot" of the size $L_{0}$ around the central magnetic field line. Coordinate $x$ is in $\theta$ direction, $y$ is in $\varphi$ direction, arbitrary units.

For $R_{0}=5 \times 10^{6} \mathrm{~cm}, \omega=10^{10} \mathrm{~s}^{-1}$, and $\gamma_{\mathrm{b}}=500$ this condition is satisfied. Lower frequencies decouple at larger distances, since $\omega_{\mathrm{p}}^{2} \propto R^{-3}$, so that $R_{0} \propto \omega^{-2 / 3}$ and $\omega R_{0} \propto \omega^{1 / 3}$. This dependence is weak so that one can expect that the condition would be satisfied in the whole emission band. If, however, the condition 5 is satisfied only marginally, at lower frequencies the emission may have the features of radiation coming from a single coherent source, thus being more polarized. At the emission site the rays start symmetrically around the magnetic field line. The model suggests that only one linearly polarized mode is generated for each propagation direction (wavevector $\boldsymbol{k}$ ). The excited mode has the electric field vector in the $\boldsymbol{k}-\boldsymbol{B}$ plane, that is, its observed polarization is determined by the projection of the magnetic field vector on the plane perpendicular to the ray propagation direction. Figure 1 shows (not to scale, arbitrary units) the above described "spot" of the size $L_{0}$ around a magnetic field line. We consider the parallel rays emitted from different points (where different magnetic field lines cross the emitting area) of the "spot" in the direction determined by the central magnetic field line. Polarization of the wave starting to propagate along one of these rays is determined by the projection of the magnetic field vector on the corresponding field line onto the plane perpendicular to the central line (shown by arrows in the figure). Since these projections are distributed symmetrically (see Fig. 1) and the emission power is expected to change weakly across this region, the combined emission (from all the rays) starting to propagate in any direction can be expected to be almost unpolarized at the emission site where the waves are generated. The combined polarization, however, will change when propagating outward from the magnetosphere to an observer.
In the simplest cold plasma case and infinite magnetic field approximation (see, e.g. Melrose 1973, 1982; Gedalin et al. 2002b) the dielectric tensor has the form

$\epsilon_{i j}=\delta_{i j}-\sigma b_{i} b_{j}$,

where $\hat{b}$ is the unit vector in the direction of the external magnetic field, and

$\sigma=\frac{\omega_{\mathrm{p}}^{2}}{\omega^{2} \gamma^{3}(1-\beta \cos \Theta)^{2}}$.

Here $\cos \Theta=\hat{\boldsymbol{b}} \cdot \hat{\boldsymbol{k}}$. It is easily seen that for electromagnetic waves

$\Delta k \approx \frac{2 \omega_{\mathrm{p}}^{2} \gamma \Theta^{2}}{\omega c\left(\gamma^{2} \Theta^{2}+1\right)^{2}}$

for $\Theta \ll 1 / \gamma$ and negligible for larger angles of propagation. The local rate of variation in $\Delta n=c \Delta k / \omega$ can be estimated as $\sim 1 / R_{\mathrm{c}}$, where $R_{\mathrm{c}}$ is the radius of the curvature of the magnetic field lines and is of the order of the neutron star radius $R_{\mathrm{c}} \sim R$. Thus, substantial mode coupling due to the field line twisting begins at

$Q=\frac{2 R \omega_{\mathrm{p}}^{2} \gamma \Theta^{2}}{\omega c\left(\gamma^{2} \Theta^{2}+1\right)^{2}} \lesssim 1$.

On the other hand

$\Delta \psi \approx \int \Delta k \mathrm{~d} l \sim \frac{2 \omega_{\mathrm{p}}^{2} \gamma \Theta^{2} R}{\omega c \gamma\left(\gamma^{2} \Theta^{2}+1\right)^{2}}$,

where the integral is taken along the ray path and the effective length of the region where the integrand in nonzero is $\sim R \Theta_{\max } \sim R / \gamma$. The above estimates are illustrated more quantitatively in Appendix A.

Let $R_{0}$ be the radius of the emission zone, where $\omega_{\mathrm{p}}^{2} \gamma \sim \omega^{2}$. Then, since $\omega_{\mathrm{p}}^{2} \propto R^{-3}$, one has

$(\gamma Q)^{-1}=\Delta \psi=\frac{\Theta^{2}}{\gamma\left(\gamma^{2} \Theta^{2} / 2+1\right)^{2}}\left(\frac{R_{0}}{R}\right)^{2}\left(\frac{\omega R_{0}}{c}\right)$.

The above calculations take into account that the rays are nearly radial. It is seen that the condition $\Delta \psi=1$ is satisfied while $Q \ll 1$. Thus, the polarization effectively stops following the magnetic field pattern well before the strong mode coupling sets in. Therefore, the PLR will be determined by the condition $\Delta \psi=1$ and the observed polarization will be the one with which the waves leave this surface. Mode coupling does not alter the polarization and does not have to be taken into account. In the course of our derivation we did not take into account possible deviations in the magnetic field from a dipole field geometry. Presence of higher multipoles might make the magnetic field line twisting stronger thus bringing the strong mode coupling onset closer to the emission region. This question is beyond the scope of the present paper and will be considered elsewhere.

For illustration of the above we use the following parameters: the pulsar period $P=1 \mathrm{~s}, R_{0}=5 \times 10^{6} \mathrm{~cm}, \gamma_{\mathrm{b}}=500$, $\gamma=50, \omega=10^{10} \mathrm{~s}^{-1}$, the angle between the rotation axis and magnetic axis $\alpha=80^{\circ}$. For these parameters the light-cylinder 

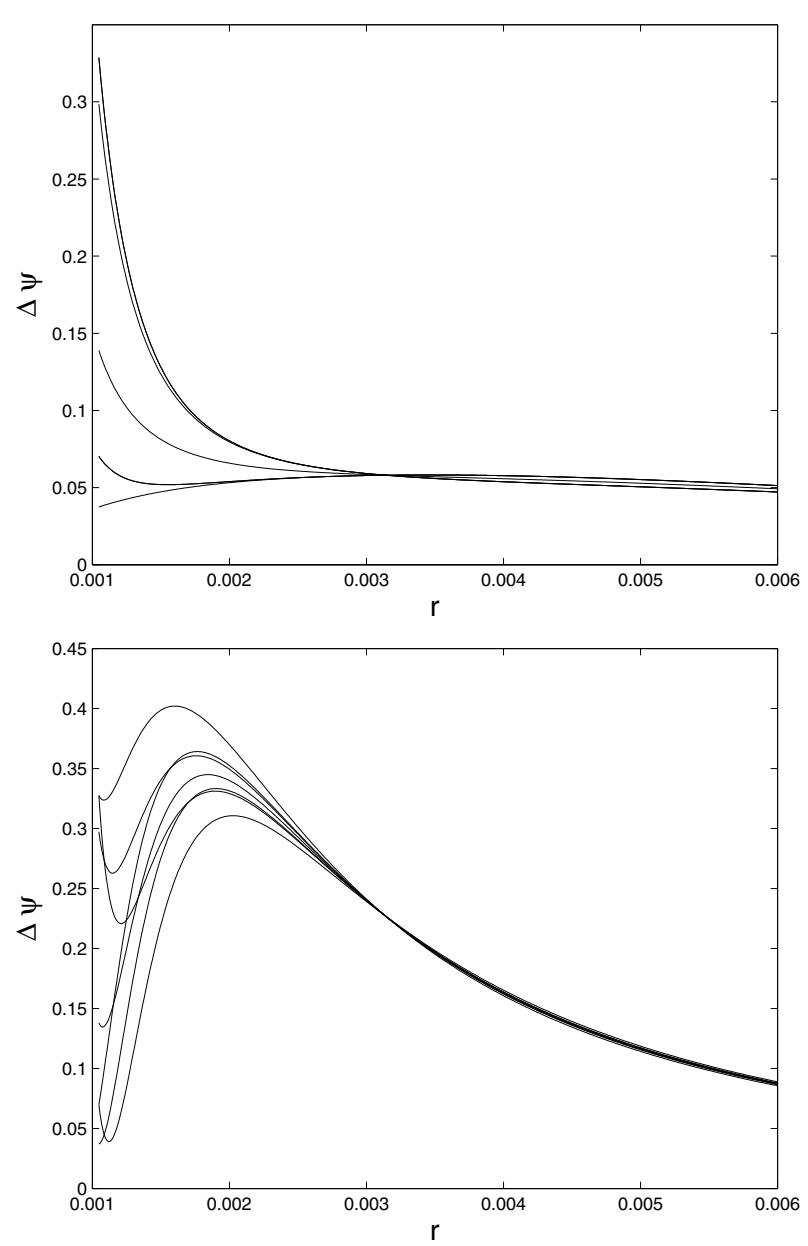

Fig. 2. Behavior of $\Delta \psi$ along the ray when the line of sight points toward the magnetic pole, $\theta=80^{\circ}, \varphi=0$ (top panel) and when the line of sight does not point exactly toward the magnetic pole, $\theta=79^{\circ}$, $\varphi=0$ (bottom panel). See the initial conditions in the text.

radius $R_{L}=c P / 2 \pi \approx 5 \times 10^{9} \mathrm{~cm}$, so that the dimensionless $r_{0}=R_{0} / R_{L} \approx 10^{-3}$. The angle $\Theta$ also depends on $R$.

Figure 2 shows several examples of the behavior of the parameter $\Delta \psi$ along the ray for different initial conditions within the angle $\approx 1 / \gamma_{\mathrm{b}}$ around the magnetic field line. The left panel is for the case when our line of sight is pointing directly to the magnetic axis $\left(\theta=80^{\circ}, \varphi=0\right)$. It is seen that the parameter $\Delta \psi$ drops very quickly as the rays propagate outward, so that one may consider the polarization as nearly fixed already at the radius $\approx 2 R_{0} \rightarrow r \approx 0.002$ (and in some cases even near the emission site). The right panel shows the behavior of the parameter $\Delta \psi$ when the line of sight does not point exactly toward the magnetic pole $\left(\theta=79^{\circ}, \varphi=0\right)$. In this case the polarization becomes fixed much further from the pulsar.

Figure 3 shows the projections of the magnetic field vector onto the plane perpendicular to the line of sight at the radius $r=0.002$. The line of sight passes through the pole which corresponds to the behavior of Fig. 2. The projections show the polarization vectors at the PLR. It is clear that the combined radiation is almost unpolarized.

Figure 4 shows the projections (polarization) for the rays starting slightly off the central field line, within angle $1 / \gamma_{\mathrm{b}}$,

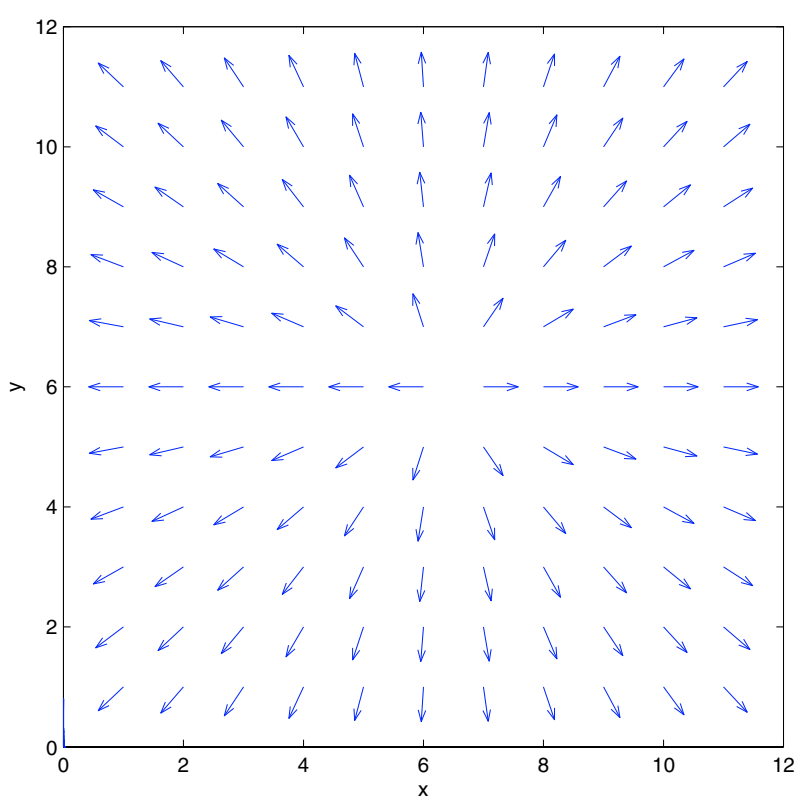

Fig. 3. Projections of the magnetic field onto the plane perpendicular to the line of sight $\left(\theta=80^{\circ}, \varphi=0\right)$ at the radius $r=0.002$. Coordinate $x$ is in $\theta$ direction, $y$ is in $\varphi$ direction, arbitrary units.

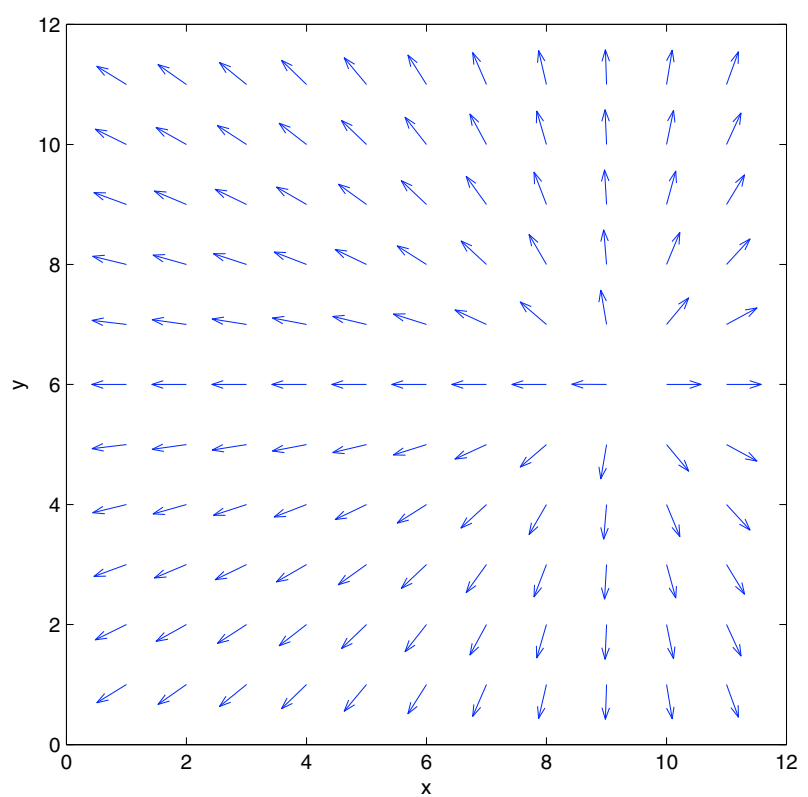

Fig. 4. Projections of the magnetic field onto the plane perpendicular to the line of sight $\left(\theta=80^{\circ}, \varphi=0\right)$ at the radius $r=0.004$. Coordinate $x$ is in $\theta$ direction, $y$ is in $\varphi$ direction, arbitrary units.

when the PRL is further from the pulsar. The preferred polarization is clearly seen, which means that one can expect substantial linear polarization (see below) of the radio waves. Finally, Fig. 5 shows the polarization pattern for the line of sight pointing toward $\theta=78^{\circ}$ and $\varphi=2^{\circ}$ (the magnetic pole corresponds to $\theta=80^{\circ}$ and $\varphi=0$ ). The emission is completely linearly polarized.

The uncertainty in our knowledge of the pulsar and plasma parameters does not allow to provide fully quantitative predictions at this stage. The presented figures illustrate the process of acquiring linear polarization on an average over many emission 


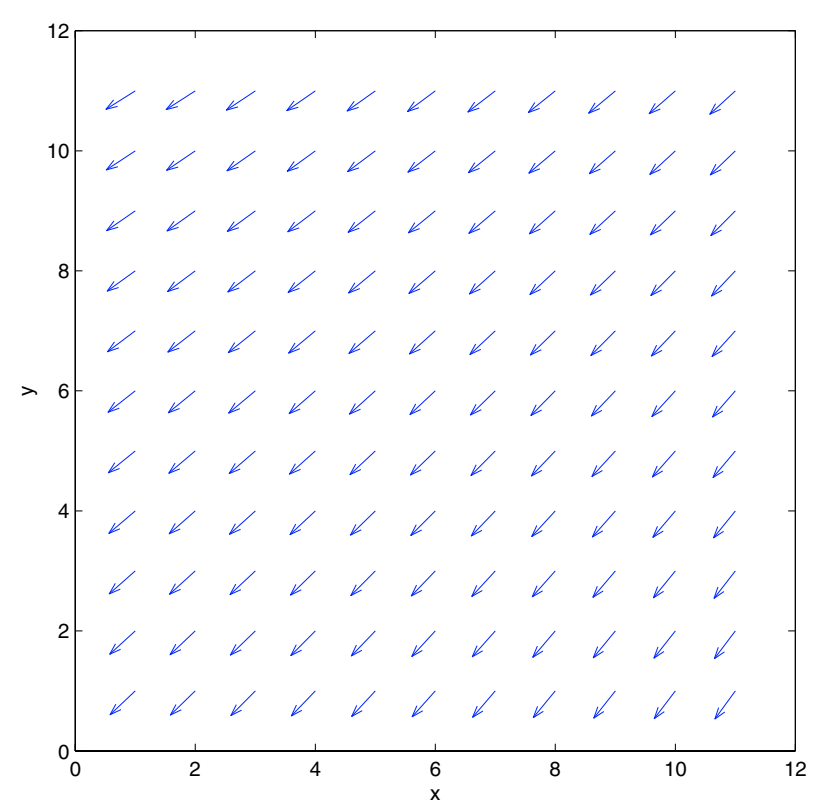

Fig. 5. Projections of the magnetic field onto the plane perpendicular to the line of sight for $\theta=78^{\circ}, \varphi=2^{\circ}$. Coordinate $x$ is in $\theta$ direction, $y$ is in $\varphi$ direction, arbitrary units.

sub-sources, due to the development of the preferential polarization in the viewed area at PLR. Our calculations show that the PLR occurs deep in the magnetosphere where the magnetic field is strong. Finally, we speculate that partial coherence of the waves generated in the emission zone could result in the appearance of circular polarization at the PLR, since the path lengths are different and waves acquire different phases on their way from the emitting radius to the PLR.

\section{Conclusions}

We have demonstrated that it is possible for the observed pulsar radiation to originate from many small sub-sources. Such radiation naturally acquires linear polarization while propagating outward due to the magnetic field geometry. The resulting polarization could depend, in general, on the mode coupling in the region where the polarization is formed. In the model where the waves are produced deep in the magnetosphere, the mode coupling is inefficient at the PLR beyond which the radiation propagation is no longer affected by the plasma. As a result, the observed polarization should be determined by the normal mode polarization at the PLR and statistical averaging over the ensemble of many sub-sources. At this stage our analysis allows only qualitative comparison with observations since details of the magnetic field structure and plasma distribution are unknown. We have not taken into account possible higher magnetic multi-poles as well as the effects of pulsar rotation. A more thorough consideration would require also a more careful treatment of wave propagation, in particular, refraction of the rays near the emission zone. Cyclotron effects (see, e.g. Melrose \& Luo 2004) may be significant in the outer magnetosphere and may eventually modify the wave polarization, although we do not expect substantial changes.
Acknowledgements. This research was supported in part by Israel Science Foundation under grant No. 170/00-1. M.G. is grateful to D. B. Melrose for illuminating discussions.

\section{Appendix A: Illustrative model solution}

We consider the emission model proposed by Gedalin et al. (2002a,b), where one linearly polarized mode is generated in the deep pulsar magnetosphere where the magnetic field is effectively infinite. For simplicity we neglect the magnetosphere rotation and use the approximation $B \rightarrow \infty$. It is instructive to derive the equations describing the behavior of the wave electric field along the ray, instead of directly using the parameters $Q$ and $\Delta \psi$. We consider the wave propagation well above the emission zone where $\omega \gg \omega_{\mathrm{p}} \sqrt{\gamma}$ and the two propagating modes are nearly transverse electromagnetic waves. Denoting the propagation direction $\hat{\boldsymbol{k}}$, the magnetic field direction $\hat{\boldsymbol{b}}$, the coordinate along the ray $z$, and the wave frequency $\omega$, one can easily arrive at the following equations:

$$
\begin{aligned}
& \hat{\boldsymbol{k}} \times \frac{\mathrm{d}}{\mathrm{d} z} \boldsymbol{E}=\frac{\mathrm{i} \omega}{c} \boldsymbol{B}, \\
& \hat{\boldsymbol{k}} \times \frac{\mathrm{d}}{\mathrm{d} z} \boldsymbol{B}=-\frac{\mathrm{i} \omega}{c} \boldsymbol{E}+\frac{4 \pi}{c} \boldsymbol{j}_{\perp},
\end{aligned}
$$

where $\perp$ refers to the propagation direction and the current has to be calculated from some plasma model. In the simplest cold plasma case and infinite magnetic field approximation one has (see, e.g. Gedalin et al. 2002b)

$4 \pi \boldsymbol{j}_{\perp}=\frac{i \omega_{\mathrm{p}}^{2}}{\omega \gamma^{3}(1-\beta \cos \Theta)^{2}} \hat{\boldsymbol{b}}_{\perp}(\boldsymbol{E} \cdot \hat{\boldsymbol{b}})$,

where $\gamma=\left(1-\beta^{2}\right)^{-1 / 2}$ and $\cos \Theta=\hat{\boldsymbol{b}} \cdot \hat{\boldsymbol{k}}$. Combining all the above one has

$\frac{\mathrm{i} c}{\omega} \frac{\mathrm{d}^{2}}{\mathrm{~d} z^{2}} \boldsymbol{E}+\frac{\mathrm{i} \omega}{c} \boldsymbol{E}=\frac{\mathrm{i} \sigma \omega}{c} \hat{\boldsymbol{b}}_{\perp}(\boldsymbol{E} \cdot \hat{\boldsymbol{b}})$,

where

$\sigma=\frac{\omega_{\mathrm{p}}^{2}}{\omega^{2} \gamma^{3}(1-\beta \cos \Theta)^{2}}$.

Using the fact that the plasma changes the refractive index by a small addition, we write $\boldsymbol{E}=\boldsymbol{E}^{\prime}(z) \exp (\mathrm{i} \omega z / c)$, where $\boldsymbol{E}^{\prime}$ depends on $z$ weakly, $(\mathrm{d} / \mathrm{d} z) E^{\prime} \ll(\omega / c) E^{\prime}$. Then one has

$-2 \frac{\mathrm{d}}{\mathrm{d} z} \boldsymbol{E}^{\prime}=\frac{\mathrm{i} \sigma \omega}{c} \hat{\boldsymbol{b}}_{\perp}\left(\boldsymbol{E}^{\prime} \cdot \hat{\boldsymbol{b}}\right)$,

or, introducing $Z=\omega z / 2 c, b_{x}=b_{\perp} \cos \xi$, and $b_{y}=b_{\perp} \sin \xi$, and defining

$E_{1}=E_{x}^{\prime} \cos \xi+E_{y}^{\prime} \sin \xi, \quad E_{2}=-E_{x}^{\prime} \sin \xi+E_{y}^{\prime} \cos \xi$

one finally gets

$\frac{\mathrm{d}}{\mathrm{d} Z} E_{1}=s E_{2}-\mathrm{i} l E_{1}$,

$\frac{\mathrm{d}}{\mathrm{d} Z} E_{2}=-s E_{1}$,

where $s=(\mathrm{d} \xi / \mathrm{d} Z)$ and $l=\sigma b_{\perp}^{2}$, Here $b_{\perp}=|\hat{\boldsymbol{b}} \times \hat{\boldsymbol{n}}|$ describes the deviation of the propagation from the parallel one, while $\xi$ 
is the angle of rotation of the perpendicular component of the magnetic field around the propagation direction (twisting).

Let us consider (A.8) and (A.9) where $l=$ const. everywhere while $s=$ const. $\neq 0$ only inside the mode coupling region $0<Z<D$ (MCR) and is zero outside. Within the framework of the pulsar magnetosphere model that would mean, for example, that the magnetic field rotates along the direction of propagation so that the angle between $\boldsymbol{k}$ and $\hat{\boldsymbol{b}}$ remains the same. Therefore, we ignore the plasma density changes across the analyzed region. At $Z=0$ and $Z=D$ the components $E_{x}$ and $E_{y}$ must be continuous. Since $\xi=\int s \mathrm{~d} Z$ is continuous too, both $E_{1}$ and $E_{2}$ are also continuous. Let us assume that these are known at $Z=0$. We are interested in the relation of, say, $\left|E_{2} / E_{1}\right|$ at $Z=D$ to $A=\left|E_{2} / E_{1}\right|$ at $Z=0$. In the transition region the solution is $E \propto \exp (\mathrm{i} p Z)$, with

$p_{1,2}=m \pm n=\frac{1}{2}\left(-l \pm \sqrt{l^{2}+4 s^{2}}\right)$,

$|m| \leq n, s \leq n, l \leq n$. Eventually we have

$\left|\frac{E_{2}}{E_{1}}\right|=\left|\frac{s\left(p_{2} A-\mathrm{i} s\right) \mathrm{e}^{\mathrm{i} p_{1} D}-s\left(p_{1} A-\mathrm{i} s\right) \mathrm{e}^{\mathrm{i} p_{2} D}}{p_{1}\left(p_{2} A-\mathrm{i} s\right) \mathrm{e}^{\mathrm{i} p_{1} D}-p_{2}\left(p_{1} A-\mathrm{i} s\right) \mathrm{e}^{\mathrm{i} p_{2} D}}\right|$.

It is instructive to analyze the case $A=0$, that is, there is only mode $E_{1}$ at the entry into the field line twisting, $s \neq 0$, region. Then

$\left|\frac{E_{2}}{E_{1}}\right|=\left|\frac{s \tan (n D)}{m \tan (n D)-\mathrm{i} n}\right|$.

If $s=0$ (no twisting) then $m \neq 0$ and $n \neq 0$, and $\left|E_{2} / E_{1}\right|=0$, that is, the radiation remains in the same mode, as it should. If $l=0(\sigma=0$, vacuum case $)$, then $m=0, n=s$, so that $\left|E_{2} / E_{1}\right|=|\tan (s D)|$. Taking into account that $s D$ is the twisting angle of the magnetic field, we see that $E_{x}$ and $E_{y}$ do not change (which is obvious).

If $n D \gg 1$ the polarization change is sensitive to the length of the MCR. If $n D \ll 1$ one gets $\left|E_{2} / E_{1}\right|=|s D /(m D+1)| \ll 1$, so that the polarization is locked (no longer changes) at the entry to the MCR.

It is useful to represent (A.12) as a two-parametric function, introducing $x=2 s / l$ and $y=l D / 2$, so that $m=-l / 2$ and $n=(l / 2) \sqrt{1+x^{2}}$, and:

$R_{1}=\left|\frac{E_{2}}{E_{1}}\right|=\frac{\left|x \tan \left(y \sqrt{1+x^{2}}\right)\right|}{\left[\tan ^{2}\left(y \sqrt{1+x^{2}}\right)+\left(1+x^{2}\right)\right]^{1 / 2}}$.

The parameter $R_{1}$ is a measure of the polarization vector rotation. If the polarization essentially follows the local normal mode polarization, $R_{1}$ should be small. The ratio $R_{2}=$ $R_{1} /|\tan (s D)|$ shows the efficiency of the polarization locking: $R_{2}=1$ corresponds to complete locking. In terms of the mode coupling parameters, $Q \approx x$ and $\Delta \psi \approx y$.

Figure A. 1 shows both measures. The left panel is for $R_{1}$ showing the decreasing efficiency of polarization dragging for increasing $x$. The right panel is for $R_{2}$ showing efficient locking for low $y$ independently of $x$.
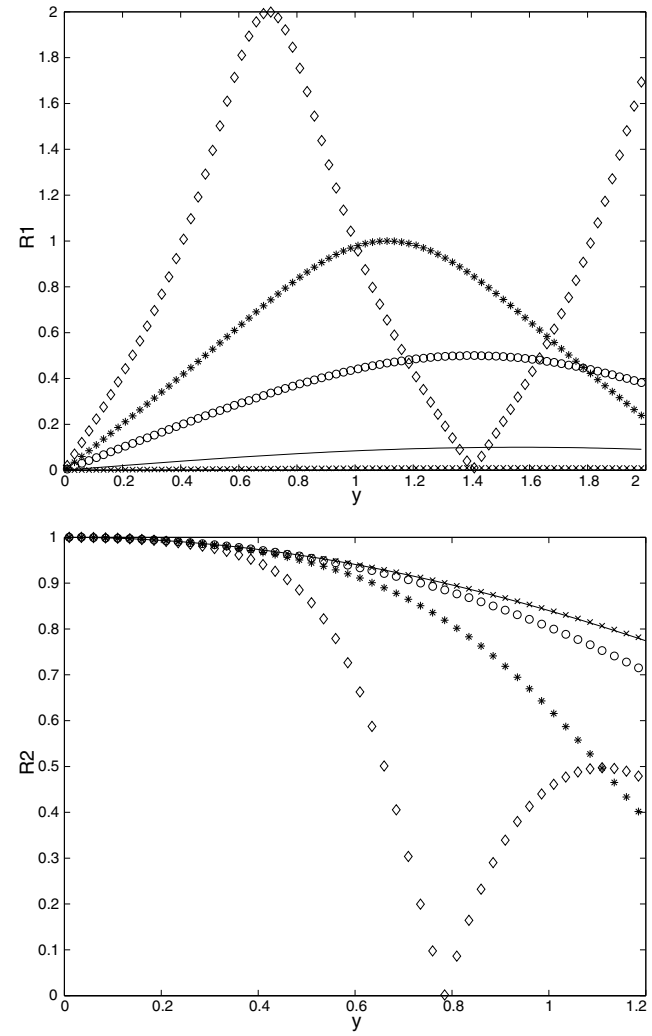

Fig. A.1. Polarization vector rotation measure $R_{1}$ (top) and polarization locking measure $R_{2}$ (bottom) for various values of $x: x=0.1$ (solid line), $x=0.5$ (circles), $x=1$ (stars), $x=2$ (diamonds), and $x=0.01$ (crosses).

\section{References}

Barnard, J. J., \& Arons, J. 1986, ApJ, 302, 138

Cheng, A. F., \& Ruderman, M. A. 1979, ApJ, 229, 348

Cordes, J. M., Rankin, J., \& Backer, D. C. 1978, ApJ, 223, 961

Gangadhara, R. T., Xilouris, K. M., von Hoensbroech, A., et al. 1999, A\&A, 342, 474

Gedalin, M., Gruman, E., \& Melrose, D. B. 2002a, Phys. Rev. Lett., 88,121101

Gedalin, M., Gruman, E., \& Melrose, D. B. 2002b, MNRAS, 337, 422

Karastergiou, A., von Hoensbroech, A., Kramer, M., et al. 2001, A\&A, 379,270

Karastergiou, A., Kramer, M., Johnston, S., et al. 2002, A\&A, 391, 247

Kramer, M., Johnston, S., \& van Straten, W. 2002, MNRAS, 334, 523 Luo, Q. 2004, MNRAS, 352, 1208

Lyubarskii, Y. E., \& Petrova, S. A. 1998, A\&A, 333, 181

Melrose, D. B. 1973, Plasma Phys., 15, 99

Melrose, D. B. 1982, Aust. J. Phys., 35, 41

Melrose, D. B., \& Luo, Q. 2004, Phys. Rev. E, 70, 016404

Melrose, D. B., \& Stoneham, R. J. 1977, Proc. Astron. Soc. Australia, 3,120

McKinnon, M. M., \& Stinebring, D. R. 1998, ApJ, 502, 883

McKinnon, M. M. 2003, ApJ, 590, 1026

Petrova, S. A. 2001, A\&A, 378, 883

Stinebring, D. R., Cordes, J. M., Rankin, J. M., et al. 1984a, ApJS, 55, 247

Stinebring, D. R., Cordes, J. M., Rankin, J. M., et al. 1984b, ApJS, 55, 279 